\title{
Adaptive Role or Glial Cell Line-Derived Neurotrophic Factor in Cerebral ischemia
}

DOI: $10.17691 / \mathrm{stm} 2017.9 .1 .08$

Received February 17, 2016

E. V. Mitroshina, PhD, Senior Researcher, Molecular Cellular Technologies Department,

Central Scientific Research Laboratory'; Associate Professor, Department of Neurotechnologies,

Institute of Biology and Biomedicine2;

B.Zh. Abogessimengane, Phb Student, Department of Neurotechnologies, Institute of Biology and Biomedicine2;

M.D. Urazov, PhD Student, Department of Neurotechnologies, Institute of Biology and Biomedicine ${ }^{2}$;

1. Hamraoui, PhD Student, Department of Neurotechnologies, Institute of Biology and Biomedicine';

T.A. Mishchenko, Senior Researcher, Molecular Cellular Technologies Department, Central Scientific

Research Laboratory'; Senior Researcher, Institute of Biology and Biomedicine;

T.A. Astrakhanova, PhD Student, Department of Neurotechnologies, Institute of Biology and Biomedicine2;

N.A. Shchelchkova, PhD, Head of Molecular Cellular Technologies Department, Central Scientific Research Laboratory';

R.D. Lapshin, PhD, Head Experimental Modeling Department, Central Scientific Research Laboratory';

T.V. Shishkina, PhD Student, Department of Neurotechnologies, Institute of Biology and Biomedicine;

I.I. Belousova, Researcher, Experimental Modeling Department, Central Scientific Research Laboratory';

I.V. Mukhina, DSc, Professor, Head of Central Scientific Research Laboratory'; Head of the Department

of Normal Physiology named after N.Y. Belenkov'; Professor, Department of Neurotechnologies,

Institute of Biology and Biomedicine'; Head of the Center of Translational Technology2;

M.V. Vedunova, DSc, Senior Researcher, Institute of Biology and Biomedicine; Associate Professor,

Department of Neurotechnologies, Institute of Biology and Biomedicine; Director, Institute of Biology and Biomedicine²

${ }^{1}$ Nizhny Novgorod State Medical Academy, 10/1 Minin and Pozharsky Square, Nizhny Novgorod, 603005,

Russian Federation;

2Lobachevsky State University of Nizhni Novgorod, 23 Prospekt Gagarina, Nizhny Novgorod, 603950,

Russian Federation

The aim of the investigation was to study the effect of glial cell line-derived neurotrophic factor (GDNF) on animals' resistance to cerebral-ischemia-induced damage.

Materials and Methods. In vivo studies were carried out on $\mathrm{C} 3 \mathrm{H}$ male mice weighing 18-40 g. Ischemia modeling was performed by bilateral irreversible occlusion of both carotid arteries. A neurological status as well as an orientative-exploratory behavior of experimental animals and their learning capability in the post-ischemic period were analyzed by using "Open field" and "Passive avoidance" tests. In addition, high-resolution respirometer Oxygraph-2k (Oroboros, Austria) was applied to study an oxygen uptake rate of brain mitochondria in ischemic conditions.

Results. GDNF application in bilateral occlusion of carotid arteries was found to contribute to the neurological status recovery. Moreover, it normalizes oxygen uptake rate of mitochondria in the post-ischemic period.

Conclusion. GDNF has a marked neuroprotective and antihypoxic effect under ischemia modeling in vivo.

Key words: glial cell line-derived neurotrophic factor; GDNF; neuroprotection; cerebral ischemia.

During several years the number of ischemic brain injury incidents has been dramatically increased, that determines topical and socially important issue for modern neurology and neuroscience. The consequences of cerebral ischemia are directly related to memory and neurological status deteriorations as well as to impairment of learning capabilities and cognitive functions. Therefore, the development of modern techniques to effectively protect the nervous system from those damaging effects is urgently needed. A promising approach to improve adaptive capabilities of nervous system supposed to the activation of endogenous systems promoting the survival of nervous cells under stress factors and maintenance their functional activity. The use of neurotrophic factors such as glial cell linederived neurotrophic factor (GDNF) is of special interest. These proteins are attracted of scientists' attention because of their possibilities to regulate neurogenesis and the functioning of the nervous cells not only in early ontogenesis, but also in an adult brain. Moreover, they are involved in the processes of synapses formation, and have a pronounced effect on growth

For contacts: Elena V. Mitroshina, e-mail: Helenmitroshina@gmail.com 
and reconstruction of axons and dendrites of cortical and hippocampal neurons [1, 2]. GDNF is produced by nervous cells for maintaining the viability of neurons under stress conditions. Its application into cultural medium contributes to maintenance and recovery of neural network functional activity in the ischemic damage in vitro $[1,3]$. Therefore, the use of synthetic analogues of neurotrophic factors supposed to open new prospects for the correction of neurodegenerative diseases and brain dysfunctions of such etiology as ischemia and traumatic injuries [4].

Hypoxia is considered as the main factor of brain cells damaging during ischemia. A decrease of oxygen concentration causes the impairment of oxidative phosphorylation on a mitochondrial membrane, the respiratory chain components separation and results in the activation of free radical processes that leads to cell death. The maintenance of mitochondrial functional state in the post-ischemic period is of great importance.

Currently, most in vivo studies are carried out on such models as middle cerebral artery occlusion or a threadocclusion method by nylon strand introduction into the middle cerebral artery [5-7]. These models are able to create a focal ischemic injury. In the present study, we used a total brain ischemia model realized by irreversible double-sided occlusion of carotid arteries. Our emphasis was laid on searching of a technique directed to effective protection of brain cells from total ischemia. Moreover, nowadays the detection of individual peculiarities of experimental animal lines to ischemic influence is of special interest, since it will enable to develop adequate models of various pathologies.

The aim of the investigation was to study the effect of glial cell line-derived neurotrophic factor on animals' resistance to cerebral-ischemia-induced damage.

It should especially notice that currently a prevailing part of the research is conducted in vitro. The existing in vivo studies have been performed mainly on rats.

Materials and Methods. To study the resistance of various mice lines to ischemia, in vivo experiments were carried out on $\mathrm{C} 3 \mathrm{H}(n=10), \mathrm{CBA}(n=10), \mathrm{SHK}$ $(n=10)$ and C57BL/6 $(n=10)$ mice weighing 28-36 g. The investigation of GDNF effect on animals' resistance to cerebral-ischemia-induced damage was performed on $173 \mathrm{C} 3 \mathrm{H}$ male mice. The main rules of keeping and caring for experimental animals corresponded to the norms provided in the order of the Ministry of Health of the Russian Federation No.708n dated 23.08.2010 "Concerning Approval of the Rules for Laboratory Practice in Russian Federation" and the ethic principles established by European Convention for the Protection of Vertebrate Animals used for Experimental and other Scientific Purposes (adopted in 18.03.1986 in Strasburg and approved in 15.06.2006 in Strasburg) and approved by the Ethics Committee of Nizhny Novgorod State Medical Academy.

According to the previous studies, a low dose of GDNF realized the most effective action [8], therefore, in current research we chose 4 and $0.4 \mu \mathrm{g} / \mathrm{kg}$ of GDNF as tested doses.

The $\mathrm{C} 3 \mathrm{H}$ animals were divided into the following groups: group 1 - intact animals $(n=16)$; group 2 sham-operated animals undergoing tissues incision and searching of arteries without their occlusion $(n=16)$; group 3 - control animals with bilateral carotid occlusion without GDNF injection ( $n=61)$; group 4 - the animals with bilateral carotid occlusion, and intranasal GDNF $(0.4 \mu \mathrm{g} / \mathrm{kg})$ introduction injected $45 \mathrm{~min}$ before the surgery and then daily within 3 postoperative days $(n=38)$; group 5 - the animals with bilateral carotid occlusion, and GDNF (4 $\mu \mathrm{g} / \mathrm{kg})$ intranasal introduction injected 45 min before the surgery and then daily within 3 postoperative days $(n=42)$. The choice of the doses was reasoned by the previous study findings [8]. Ischemia was simulated in the mice from CBA, SHK and C57BL/6 lines were subjected to ischemia without tested substances introduction.

A brain ischemia model. In order to induce ischemic damage in different brain structures we used a model of bilateral carotid occlusion. The animals were anesthetized by pentobarbital in concentration of $70 \mathrm{mg} / \mathrm{kg}$. To eliminate the influence of circadian rhythms, the experiment was carried out at one and the same time of day. The anterior neck surface was shaved off followed by soft tissue incision. In a surgical area we detected the left and then the right carotid artery with subsequent simultaneous ligation of the vessels by non-absorbable ligature. The wound was then sutured and powdered by Streptocide. All procedures took 10-15 min.

Neurological status determination. To assess nervous system functional state of experimental animals after cerebral-ischemia-induced damage we determined a neurological status using the neurologic impairment assessment scale for small laboratory animals [9] and Garcia scale [10, 11] modified for mice. Neurological status was determined before ischemia modeling, within 10 days of the post-ischemic period, and on day 14 after surgery.

The neurologic impairment assessment in small laboratory animals was conducted by detection of 10 involuntary congenital behavioral responses, each response being assessed from zero to two points, where 2 points meant no response. Then the measured points were summed up.

Neurological status evaluation results were interpreted as follows:

from 10 to 20 points: severe CNS damage;

from 6 to 9 points: moderate CNS damage;

from 1 to 5 points: slight CNS damage.

According to Garcia scale of neurological status, 6 tests were used to assess the asymmetry of movements and responses of an animal in points, which were summed up. The total score assigned to each mouse after the test was the summation of the findings of all 6 tests. Minimal neurological result was 3 points (severe damage), and maximal was 18 points (no damage). 
"Open field" test. General motor and orientingexploratory activity of the experimental animals were tested before ischemia modeling, and on days 1, 7 and 14 of the post-ischemic period by using Open Field setup (OpenField LE800S; Panlab Harvard Apparatus, Spain). Behavioral responses of animals were registered by camera Sony SSC-G118 (Japan). The findings were recorded and analyzed in the Smart 3.0.03 software program (Panlab Harvard Apparatus, Spain; Stoelting, USA).

The test consists in the quantitative measurement of behavior components of animals placed in new open space. The following parameters of mice behavioral activity were recorded: horizontal motor activity including running inside the field; vertical motor activity with recording a total number of upright posture without support and with resting on a sidewall; the number and duration of grooming acts; the duration of freezing and the number of defecations and urinations characterizing an emotional level of an animal.

The formation of conditioned passive avoidance reflex. The learning capability of animals was determined according to the assessment of a latent entry time of a mouse from a light to a dark compartment of a shuttle chamber $(60 \times 20 \times 25 \mathrm{~cm})$ with an electrified grid floor, the chamber being separated by a partitionwall with a hole for two similar compartments: darkened and lightened (Shuttle Box LE918; Panlab Harvard Apparatus, Spain). The training of animals was performed once, on day 12 after ischemia modeling. An animal was placed in a light compartment, its back to the dark compartment (start position). Under the influence of exploratory behavior and congenital preference of dark areas (photophobia), mice, generally, entered a darkened compartment rather quickly. A latent period of dark compartment entry was recorded. As soon as a mouse had passed into the compartment, its paws were electrocutaneously irritated (currency $0.08 \mathrm{~mA}$ ) for $5 \mathrm{~s}$, and after that the animal was put into a house cage. On days 14 and 18 after ischemia we checked the memory trace safety. For this purpose the animals were tested in the same compartment, though without currency feed. A mouse was put in a light compartment of a chamber, and then stated a latent entry time of a mouse from a light to a dark compartment. If an animal did not pass into a dark compartment within $180 \mathrm{~s}$, its memory trace was considered to be fully preserved.

Brain mitochondria isolation. To study molecular mechanisms of neurotrophins action we isolated mitochondria from brain cells and measured their functional parameters. Mitochondria were isolated by standard differential centrifugation. All procedures were carried out on ice as well as equipment and isolation media were cooled. After decapitation, immediate trepanation was performed, the brain being removed (within no more than $20 \mathrm{~s}$ ), put in a preliminarily cooled porcelain mortar, and washed with an ice isolation medium containing the following (in $\mathrm{mM}$ ): 70 saccharose;
210 mannitol; 30 HEPES; 0.1 EDTA (pH 7.4), followed by cerebellum removal. Cerebral hemispheres and the brainstem were homogenized in an isolation medium in a glass homogenization test tube put in ice. Teflon pestle of a homogenizer, driven by an electric motor, has a clearance which preventing the mitochondrial damage. The ratio of tissue weight and isolation medium was 1:7. The brain homogenate was preliminarily centrifuged at $2,700 \mathrm{rpm}$ (temperature interval was from -3 to $0^{\circ} \mathrm{C}$, $10 \mathrm{~min}$ ). The supernatant was collected in a tube and centrifuged for $15 \mathrm{~min}$ at $8,500 \mathrm{~g}$. The precipitated mitochondria were washed with cold $\left(4^{\circ} \mathrm{C}\right)$ isolation medium, and resuspended in a medium containing (in $\mathrm{mM}$ ) 210 mannitol; 70 saccharose; 0.1 EGTA;

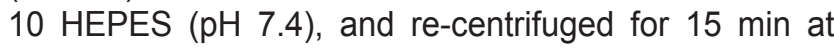
$8,500 \mathrm{~g}$. The mitochondrial suspension was kept on ice being prevented from freezing.

Brain mitochondrial respiration parameters were determined by evaluating mitochondrial oxygen uptake rate, which was recorded by a high resolution respirometer Oxygraph-2k (Oroboros, Austria) in a closed chamber volume of $2 \mathrm{ml}$ at constant stirring and controlled temperature $\left(37^{\circ} \mathrm{C}\right)$. Mitochondrial incubation medium contained the following (in $\mathrm{mM}$ ): $120 \mathrm{KCl}$; $5 \mathrm{NaH}_{2} \mathrm{PO}_{4} ; 10$ HEPES; 5 glutamate; 5 malate; $14 \mathrm{MgCl}_{2}$ $(\mathrm{pH}$ 7.4). Mitochondrial protein concentration in an oxygen chamber was $0.5 \mathrm{mg} / \mathrm{ml}$.

The functional state of mitochondrial respiration chain was assessed using the following parameters: a) V4 - high content of substrates in the incubation medium: $5 \mathrm{mM}$ glutamate and $5 \mathrm{mM}$ malate (I complex substrates) without ADP; b) V3 - the same conditions as in $\mathrm{V} 4$, with $5 \mathrm{mM} \mathrm{ADP}$ (with a respiration chain itself being a factor limiting the breathing rate). The intensity of complex II of the respiration chain was assessed after complex I inhibited by $0.5 \mu \mathrm{M}$ rotenone, and complex II stimulated by $10 \mathrm{mM}$ sodium succinate.

Statistical data analysis. All quantified data are presented as mean \pm standard error of mean (SEM). The significance of differences between the experimental groups was determined by ANOVA in the SigmaPlot 11.0 software program (Systat Software Inc., USA). The differences were considered significant if the corresponding $p$ value was less than 0.05 .

Results. First, we compared the resistance of different mice lines to ischemia damaging effect.

The effective searching of ways aiming at correction a negative effect of ischemia on nervous tissue suggests the development of convenience and adequate experimental ischemia models applied on laboratory animals. Currently, several brain ischemia models are widely used in neurobiological studies (cardiac arrest, monofilament fiber introduction into the internal carotid artery, medial cerebral artery ligation via a burr hole, photothrombosis, etc.) [12-14]. Alongside with advantages, all the models have disadvantages as well. We modified and adapted for mice the cerebral ischemia model by the irreversible occlusion of major vessels 
supplying the brain with blood - carotid arteries. Its advantage is marked extensive cerebral ischemic damage, since carotid arteries account for $70-75 \%$ cerebral blood supply. The application of the model supposed to assess the effect of various substances, presumably with neuroprotective, antihypoxic and reparative effects. The $\mathrm{C} 3 \mathrm{H}, \mathrm{CBA}, \mathrm{SHK}$ and $\mathrm{C} 57 \mathrm{BL} / 6$ mice survival and their neurological status were analyzed in the post-ischemic period. $\mathrm{C} 3 \mathrm{H}$ mice were found to show the maximum resistance to ischemia.

On day 10 after ischemia modeling the $\mathrm{C} 3 \mathrm{H}$ mice survival was $60.50 \pm 8.95 \%$, while in other experimental group this parameter was from $33.33 \pm 11.20 \%$ (CBA) to $20.20 \pm 7.55 \% \quad$ (C57BL/6) already at day 4 of the post-ischemic period. The neurological status evaluation according to both conducted tests (the neurologic impairment assessment scale in small laboratory animals and Garcia scale) showed the development of significant moderate severity of CNS damage for CBA and C57BL/6 mice, while for $\mathrm{SHK}$ and $\mathrm{C} 3 \mathrm{H}$ mice no significant impairments were revealed.

Moreover, a comparison of different mice lines on the preservation of memory traces during the formation of conditioned passive avoidance reflex was conducted. The animals of $\mathrm{C} 3 \mathrm{H}$ line were found to have the shortest latent period of passing between the compartments $(98.23 \pm 26.15 \mathrm{~s})$, and the longest period was showed for SHK line (148.82 $\pm 31.18 \mathrm{~s})$.

To assess the GDNF effect on motor and orienting-exploratory activity of experimental animals and their learning capability in a long-term postischemic period, we chose $\mathrm{C} 3 \mathrm{H}$ line mice demonstrating high survival rates after severe ischemic brain damage. We studied the effect of neurotrophin on animals' resistance to cerebralischemia-induced damage on $173 \mathrm{C} 3 \mathrm{H}$ mice weighing $25-40 \mathrm{~g}$. To exclude the influence of hormonal cycle phases on behavioral activity and learning capability, the study was conducted on male mice.

Interestingly, the survival of $\mathrm{C} 3 \mathrm{H}$ male mice after irreversible ischemia modeling was significantly $(p<0.05$; ANOVA) lower in comparison with $\mathrm{C} 3 \mathrm{H}$ female mice. This phenomenon supposed to be explained by high adaptive reserves of female organism to various stress factors.

Table 1
The resistance degree to ischemia was evaluated by animals' survival and neurological status within 10 days after ischemia modeling. GDNF application increased the mice resistance in the post-ischemic period (Table 1). The GDNF dose of $0.4 \mu \mathrm{g} / \mathrm{kg}$ has the most pronounced effect. However, the animal mortality in the "GDNF $4 \mu \mathrm{g} / \mathrm{kg}$ " group was increased after the discontinuation of GDNF maintenance injections.

The marked neurologic impairment in the modeled ischemia was observed on the first day after surgery (Figure 1). According to neurologic impairment

The GDNF effect on mice survival in irreversible brain ischemia model

\begin{tabular}{lcccc}
\hline \multirow{2}{*}{ Animal groups } & \multicolumn{4}{c}{ Animal mortality after ischemia (\%) } \\
\cline { 2 - 5 } & Day 1 & Day 5 & Day 7 & Day 10 \\
\hline Control (ischemia) & $84.2 \pm 4.3$ & $93.4 \pm 2.1$ & $94.5 \pm 2.6$ & $94.5 \pm 2.3$ \\
\hline GDNF $0.4 \mu \mathrm{g} / \mathrm{kg}$ & $77.6 \pm 3.9$ & $84.5 \pm 1.5^{*}$ & $84.0 \pm 2.5^{*}$ & $84.1 \pm 4.3$ \\
\hline GDNF $4 \mu \mathrm{g} / \mathrm{kg}$ & $81.0 \pm 4.1$ & $87.6 \pm 2.3$ & $95.3 \pm 4.3$ & $95.9 \pm 4.7$ \\
\hline
\end{tabular}

* Statistical significance with the control group, $p<0.05$; ANOVA.

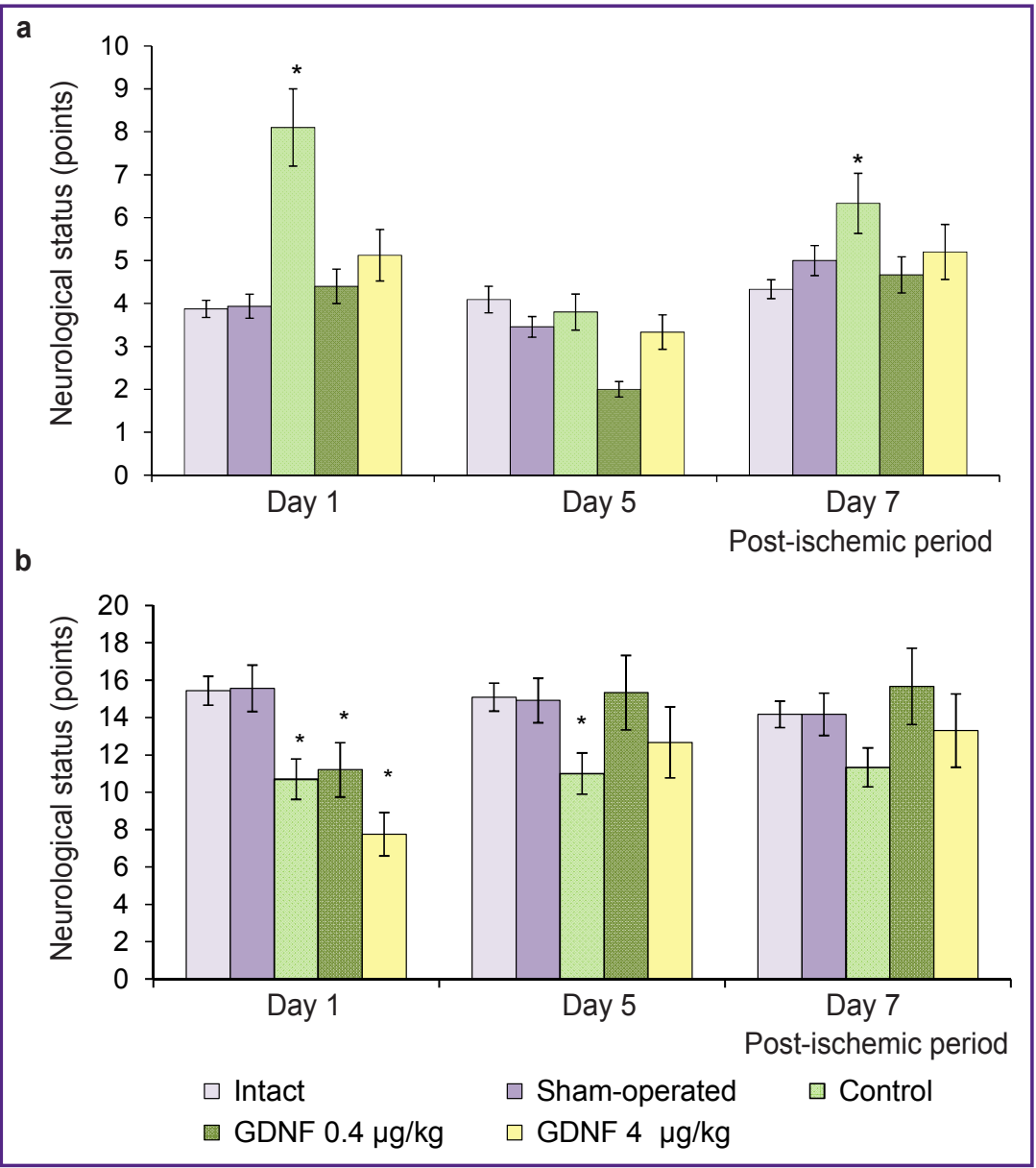

Figure 1. Neurological status of $\mathrm{C} 3 \mathrm{H}$ mice after brain ischemia modeling: (a) according to neurologic impairment assessment scale in small laboratory animals; (b) according to Garcia scale; * statistical significance with the intact group, $p<0.05$; ANOVA 
assessment scale, the significant differences between an intact and a control group on the first and seventh postoperative days were revealed. In the control group moderate and severe impairments of a neurological status were recorded on the first day after surgery: $8.10 \pm 1.21$ points. The animals had marked ptosis, disturbances in the implementation of motor and behavioral tests (coming out of a circle, moving along the crossbar, etc.). No significant differences were found between the intact group and "GDNF $0.4 \mu \mathrm{g} / \mathrm{kg}$ " and "GDNF $4 \mu \mathrm{g} / \mathrm{kg}$ " groups, the neurological deficit of the animals with GDNF treatment was moderately expressed.

The analysis of neurological status according to Garcia scale showed the significant difference between the intact and control groups (Figure 1 (b)). The animals had asymmetric motions and impaired responses in tests of spontaneous activity, body proprioception and reaction to the vibrissa touching. Neurological deficit of these animals was generally severe and moderate and accounting for $10.70 \pm 1.31$ points on day 1 after surgery. The animals with treatment by both doses of GDNF had significant decrease in the parameters characterizing their neurological status on the first postoperative day in comparison with the intact group (11.20 \pm 1.45 and $7.75 \pm 1.88$ points, respectively). In a long-term postischemic period the parameters of neurological status were normalized and had no differences from the intact group by day 5 after surgery.

Thus, GDNF contributes to animals' survival in ischemic brain model, and promotes neurological status normalization in a post-ischemic period. Low concentration of GDNF $(0.4 \mu \mathrm{g} / \mathrm{kg})$ showed the best result, that is consistent with the previously received data [8]. In this regard, further studies in a group of experimental animals with a preventive injection of GDNF $(0.4 \mu \mathrm{g} / \mathrm{kg})$ were carried out.

General motor and orienting-exploratory activity of the experimental animals were tested by using "Open field" setup on days 1, 7 and 14 of the post-ischemic period. The analysis of behavioral responses showed the time of animal freezing after total ischemia to increase by 9.8 times on the first postoperative day compared to the intact group (Table 2). The data indicate a decrease in

Table 2

Duration of animal freezing in "Open field" test after ischemia modeling

\begin{tabular}{lccc}
\hline \multirow{2}{*}{ Animal groups } & \multicolumn{3}{c}{ Freezing reaction duration after ischemia (s) } \\
\cline { 2 - 4 } & Day 1 & Day 7 & Day 14 \\
\hline Intact & $13.170 \pm 6.396$ & $15.63 \pm 8.56$ & $20.20 \pm 11.94$ \\
\hline Sham-operated & $27.96 \pm 17.37$ & $12.88 \pm 7.38$ & $26.59 \pm 17.49$ \\
\hline Control (ischemia) & $129.11 \pm 38.89^{*}$ & $6.52 \pm 1.85$ & $5.02 \pm 5.11$ \\
\hline GDNF $0.4 \mu \mathrm{g} / \mathrm{kg}$ & $3.64 \pm 2.77$ & $11.92 \pm 6.05$ & $7.95 \pm 4.18$ \\
\hline
\end{tabular}

* Statistical significance with the intact group, $p<0.05$; ANOVA.
Table 3

The study of conditioned passive avoidance reflex reproduction after ischemia modeling

\begin{tabular}{lccc|}
\hline \multirow{2}{*}{ Animal groups } & \multicolumn{3}{c}{ Latent period time after ischemia (s) } \\
\cline { 2 - 4 } & $\begin{array}{c}\text { Learning } \\
\text { (day 12) }\end{array}$ & $\begin{array}{c}\text { Reproduction 1 } \\
\text { (day 14) }\end{array}$ & $\begin{array}{c}\text { Reproduction 2 } \\
\text { (day 18) }\end{array}$ \\
\hline Intact & $72.68 \pm 24.44$ & $119.12 \pm 27.92$ & $127.53 \pm 23.20$ \\
\hline Sham-operated & $84.73 \pm 22.60$ & $141.50 \pm 22.08$ & $165.40 \pm 21.01$ \\
\hline Control (ischemia) & $68.80 \pm 20.16$ & $169.10 \pm 38.51$ & $154.73 \pm 35.37$ \\
\hline GDNF 0.4 $\mathrm{gg} / \mathrm{kg}$ & $139.4 \pm 40.6$ & $118.27 \pm 42.89$ & $104.87 \pm 37.48$ \\
\hline
\end{tabular}

exploratory activity and an increase of stress level in animals. However, these parameters were normalized by day 5 of the post-ischemic period. In the group of animals with pre- and postoperatively injection of GDNF $(0.4 \mu \mathrm{g} / \mathrm{kg})$ the duration of freezing reaction was comparable with the intact group.

The other parameters of orienting-exploratory activity (such as horizontal and vertical motor activity, the number and duration of grooming acts, the number of defecations and urinations) are not significantly differed between the experimental groups. The intranasal administration of GDNF $(0.4 \mu \mathrm{g} / \mathrm{kg})$ promotes to reduce the freezing time and normalizes animal motor activity in a post-ischemic period.

To estimate the preservation of cognitive functions and learning capabilities after cerebral-ischemia-induced damage we analyzed the maintenance of memory traces in the formation of conditioned passive avoidance reflex and its preservation (duration of a latent period). The training of animals was performed on day 12 after ischemia modeling while the reflex reproduction was measured 2 and 5 days after the training. The data concerning a duration of a latent period of transition between the light and darkened compartments are presented on Table 3. The latent period time after training increased by 1.7-2.5 times in all groups. The parameters of the experimental groups during both procedures were not significantly differed from the parameters of the intact group.

Thus, the studies demonstrated GDNF to enhance animals' resistance to ischemia. Preventive intranasal GDNF injection combined with maintaining introduction during the post-ischemic period promotes the maintenance of animals' viability, the normalization of neurological status and motor activity. The GDNF dose of $0.4 \mu \mathrm{g} / \mathrm{kg}$ has the most pronounced effect.

To study molecular mechanisms of GDNF neuroprotective effect we analyzed oxygen uptake rate of brain mitochondria on days 1 and 7 after irreversible cerebral ischemia modeling (Figure 2). The assessment of oxygen uptake rate of mitochondria regarded as a key parameter of functional state of the organelle, since it depends on the number of mitochondria as well as on 
the state of respiratory chain enzymes.

Carried out experiments revealed the oxygen uptake rate of mitochondria in the intact group amounted to $234.50 \pm 32.36 \mathrm{pmol} /(\mathrm{s} \cdot \mathrm{ml})$. Ischemia caused the decrease of oxygen uptake rate of mitochondria in 2.4 times (up to $96.47 \pm 6.64 \mathrm{pmol} /(\mathrm{s} \cdot \mathrm{ml}))$ in comparison with the intact group on day 1 of the post-ischemic period indicating the suppression of mitochondrial respiratory chain (Figure 2 (a)). The oxygen uptake rate by mitochondria was normalized by day 7 after ischemia modeling $(208.85 \pm 9.01 \mathrm{pmol} /(\mathrm{s} \cdot \mathrm{ml}))$. Our findings could be served as the evidence of post-ischemic adaptation in a long-term period, when compensatory reactions are activated resulting in an increased number of mitochondria and de novo protein synthesis.

The study of GDNF effect on oxygen metabolism in brain mitochondria showed its possibility to increase oxygen uptake rate of mitochondria in brain ischemia modeling (Figure 2 (a)). The oxygen uptake rate in this experimental group $24 \mathrm{~h}$ after cerebral-ischemia-induced damage was $187.75 \pm 40.90 \mathrm{pmol} /(\mathrm{s} \cdot \mathrm{ml})$ and no differed from the intact values.

The evaluation of respiratory chain functional state in the presence of ADP demonstrated ischemic damage to cause sharp inhibition of respiratory complex I activity. Oxygen uptake rate in the presence of ADP decreased by 6.9 times compared to the intact group $24 \mathrm{~h}$ after ischemia modeling (Figure 2 (b)). In animals injected by GDNF the respiratory chain activity decreased by 2.7 times only. It should be noted that on day 7 of the post-ischemic period in "GDNF $0.4 \mu \mathrm{g} / \mathrm{kg}$ " group the oxygen uptake rate of mitochondria in the presence of ADP was normalized, while in the control group this parameter was significantly lower in comparison with the intact animals.

The study of oxygen uptake rate of mitochondria in the work of respiratory complex II revealed significant decrease of the parameter on the first post-ischemic day (Figure 2 (c)). GDNF group showed no differences from the intact values.

Thus, GDNF maintains the efficiency of respiratory chain work and mitochondria functional state contributing to brain cell adaption to ischemia damaging effects.

Discussion. The searching of substances able to increase the resistance of animals to negative ischemia effect is assumed to consider the characteristics of

b
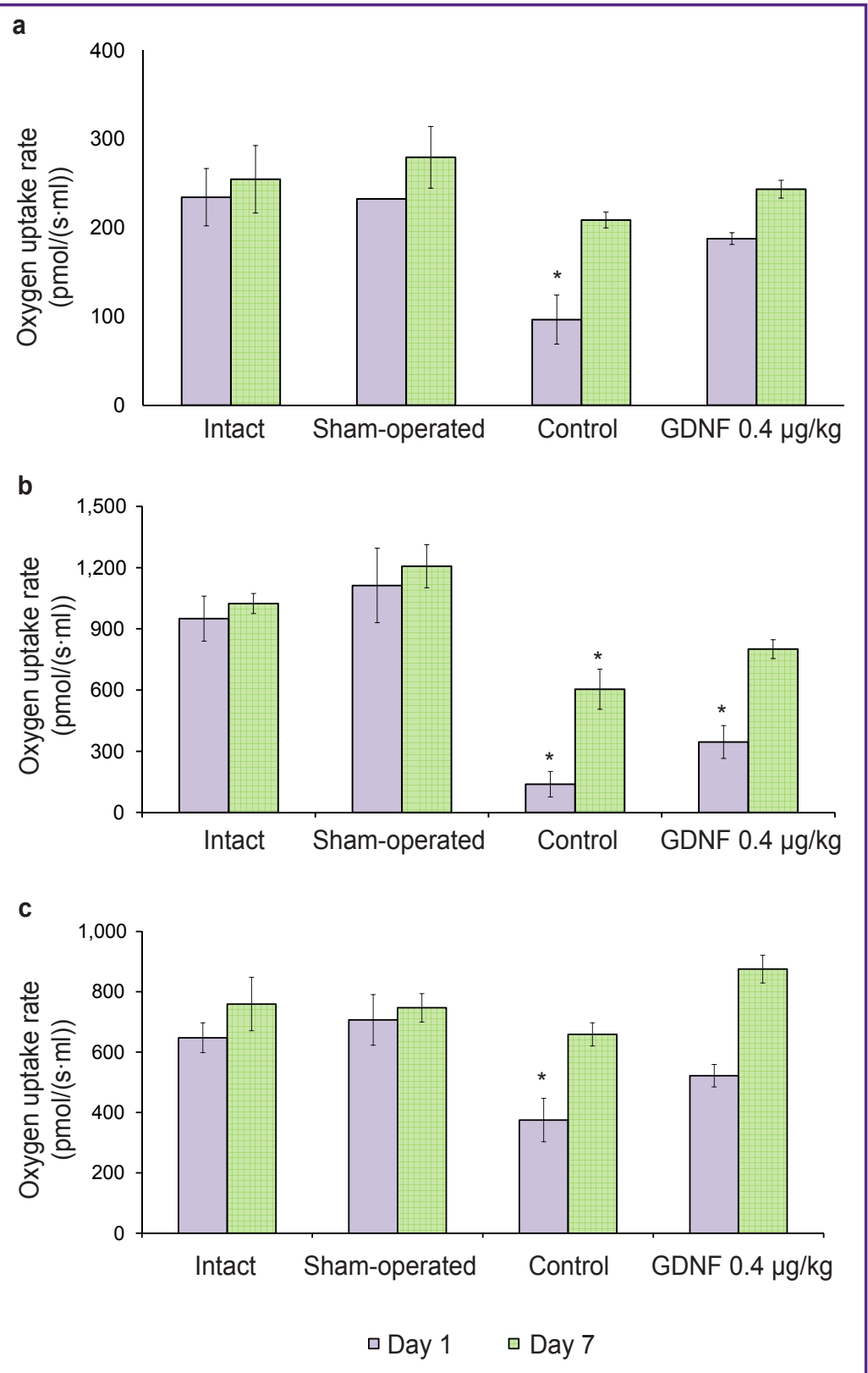

Figure 2. The change of mitochondrial oxygen uptake rate: (a) oxygen uptake rate of brain mitochondria; (b) the state of vigorous activity induced by ADP application; (c) mitochondrial oxygen uptake rate during the work of complex II in the mitochondrial respiratory chain; * significant differences with intact animals, $p<0.05$; ANOVA

sensibility to ischemic damage typical for certain animal lines. The response of different animal lines to one and the same stress effects are known to be extremely different [15-17]. Therefore, in the present study we compared the resistance of different mice lines to cerebral-ischemia-induced damage.

Nowadays, a bilateral carotid occlusion technique regarded as the most common method of total cerebral ischemia model performed on laboratory animals [13]. However, this model is difficult to realize on mice and has several peculiarities. Its disadvantage is a high variability 
of ischemic outcome [18, 19], therefore, the resistance to ischemic damage in different lines of mice represents a topical issue for neurobiological researches [17, 20, 21]. Our study demonstrated $\mathrm{C} 3 \mathrm{H}$ line to be the most resistant to ischemic damage among all tested animals (C3H, CBA, SHK and C57BL/6). One of possible reasons of such variability could be individual differences of collateral blood flow through the circle of Willis [22, 23], since even within one genotype might differ significantly in arterial cerebrovascular structures, in particular, the presence or absence of the posterior communicating artery [23]. Due to this fact, it is necessary to carry out preliminary ischemia resistance test on a certain animal line during investigation of different aspects of ischemic damages. From this point of view, particular attention is given to studying the problem of cognitive reactions of certain types of laboratory animals. $\mathrm{C} 3 \mathrm{H}$ is characterized both as the most resistant line, and also having marked peculiarities in the formation of conditioned passive avoidance reflex, which qualitatively differs this line of mice from others.

Investigation the effect of glial cell line-derived neurotrophic factor on $\mathrm{C} 3 \mathrm{H}$ mice resistance to ischemic damage showed the GDNF at doses of 0.4 and $4 \mu \mathrm{g} /$ $\mathrm{kg}$ to increase the animal survival and contribute to the normalization their neurological status and motor and orienting-exploratory activity in the postischemic period. The GDNF dose of $0.4 \mu \mathrm{g} / \mathrm{kg}$ has the most pronounced effect. Thus, GDNF exhibits strong neuroprotective and antihypoxic effect that is consistent with the previously received data. Several studies performed on focal and total ischemia models in vivo demonstrated that preventive GDNF injection or its immediate application after ischemia decreases the severity of ischemic damage [24, 25]. The mechanisms of neuroprotective effects supposed to be related with GDNF capability to partially negate of glutamate excitotoxicity induced by ischemia, and the generation of active oxygen forms, as well as inhibit apoptotic activity of caspases, activate immediate early genes and therefore increase cell adaptation to stress factors $[26,27]$. GDNF is a universal bioactive molecule able to provide the viability maintenance of certain neurons as well as to combine metabolic reactions of separate components in neuron-glial network into a single functional structure. In various focal and total cerebral ischemia experimental models it was shown that GDNF promotes cell survival, maintenance of processes, and synaptogenesis [3]. The activation of universal multicomponent GFR1 receptors is known to promote intracellular metabolic cascades preventing the activation of cell death [28-30].

An ischemic damaging effect on the organism includes a number of components, hypoxia being a key one for nervous cells. To maintain a high metabolic level, neurons constantly require a great number of highenergy compounds (ADP) that makes them sensitive to oxygen deficiency and impaired work of mitochondrial respiratory chain. In the case of oxygen concentration decrease, pathological processes in brain tissues are triggered resulting in oxidative phosphorylation disturbance, violations in the mitochondria functional state, and subsequently, the decrease in cell energy metabolism leading to the activation of free radical processes and apoptosis. A decreased number of free radicals formed in ischemia regarded as one of the mechanisms of GDNF protective action [26, 31-33]. Our findings revealed that application of GDNF in the dose of $0.4 \mu \mathrm{g} / \mathrm{kg}$ contributes to preservation of oxygen uptake rate of brain mitochondria, and maintenance normal activity of enzymatic respiratory complexes. Therefore, neuroprotective and antihypoxic effects of glial cell-derived neurotrophic factor are implemented through the influence on mitochondria functional and metabolic state. Our data are experimental basis for further studies concerning GDNF as a potential substance used for the correction of severe cerebral ischemic damage.

Conclusion. Glial cell line-derived neurotrophic factor has strong neuroprotective and antihypoxic effect. Intranasal injection of GDNF contributes to animal survival and normalizes neurological status and motor activity of mice in severe ischemia brain injury modeling. The effect implemented by GDNF is not limited to the previously described intracellular metabolic cascades. Carried out experiments showed the GDNF effect on the functional state of mitochondria.

Study Funding. The research was supported by grants from the Russian Foundation for Basic Research (No.16-04-00245, No.16-34-00301). The publication has been prepared as part of the state projects "Provision Scientific Research". The study was performed within the framework of the state task (projects 17.3335.2017/ $\Pi Ч$ and 6.6379.2017/БЧ).

Conflicts of Interest. The authors declare that there are no conflicts of interest regarding the publication of this paper.

\section{References}

1. Vedunova M.V., Shishkina T.V., Mishchenko T.A., Mitroshina E.V., Astrakhanova T.A., Pimashkin A.S., Mukhina I.V. Antihypoxic and neuroprotective effects of glial cell-derived neurotrophic factor (GDNF) in cultures of dissociated hippocampal cells under conditions of experimental hypoxia. Bull Exper Biol Med 2016; 161(1): 168-174, https:// doi.org/10.1007/s10517-016-3369-3.

2. Shishkina T.V., Vedunova M.V., Mishchenko T.A., Mukhina I.V. The role of glial cell line-derived neurotrophic factor in the functioning of the nervous system (review). Sovremennye tehnologii $v$ medicine 2015; 7(4): 211-220, https://doi.org/10.17691/stm2015.7.4.27.

3. Duarte E.P., Curcio M., Canzoniero L.M., Duarte C.B. Neuroprotection by GDNF in the ischemic brain. Growth Factors 2012; 30(4): 242-257, https://doi.org/10.3109/089771 94.2012.691478.

4. Vedunova M.V., Mishchenko T.A., Mitroshina E.V., 
Mukhina I.V. TrkB-mediated neuroprotective and antihypoxic properties of brain-derived neurotrophic factor. Oxid Med Cell Longev 2015; 2015: 1-9, https://doi. org/10.1155/2015/453901.

5. Vasilyev I.A., Stupak V.V., Chernykh V.A., Zaidman A.M., Polovnikov E.V., Chernykh E.R., Shevela E.Y., Dergilev A.P. Experimental models of vascular lesions of the brain (literature review). Uspekhi sovremennogo estestvoznaniya 2015; 1(3): 366-369.

6. Kaya A.H., Erdogan H., Tasdemiroglu E. Searching evidences of stroke in animal models: a review of discrepancies. Turk Neurosurg 2016, https://doi. org/10.5137/1019-5149.jtn.15373-15.2.

7. Kumar A., Aakriti, Gupta V. A review on animal models of stroke: an update. Brain Res Bull 2016; 122: 35-44, https:// doi.org/10.1016/j.brainresbull.2016.02.016.

8. Vedunova M.V., Sakharnova T.A., Mitroshina E.V., Shishkina T.V., Astrakhanova T.A., Mukhina I.V. Antihypoxic and neuroprotective properties of BDNF and GDNF in vitro and in vivo under hypoxic conditions. Sovremennye tehnologii $v$ medicine 2014; 6(4): 38-47.

9. Kul'chikov A.E., Makarenko A.N., Novikova Yu.L., Dobychina E.E. Sposob opredeleniya nevrologicheskogo defitsita u melkikh laboratornykh zhivotnykh pri porazhenii golovnogo mozga. A.s. 2327227 C2 [Neurologic impairment determination method in small laboratory animals with brain lesions. A.c. 2327227 C2]. 2008.

10. Garcia J.H. Early reperfusion as a rationale of therapy in ischemic stroke. Rev Neurol 1995; 23(123): 1067-1073.

11. Kuster G.W., Baruzzi A.C., Pacheco Ede P., Domingues R.B., Pieruccetti M., Giacon L.M., Garcia J.C., Furlan V., Massaro A.R. Early reperfusion therapy in acute ischemic stroke after recent myocardial infarction. Arq Neuropsiquiatr 2016; 74(8): 690-691, https://doi. org/10.1590/0004-282x20160099.

12. Manukhina E.B., Terekhina O.L., Belkina L.M., Abramochkin D.V., Budanova O.P., Mashina S.Yu., Smirin B.V., Yakunina E.B., Downey H.F. Vasoprotective effect of adaptation to hypoxia in myocardial ischemia and reperfusion injury. Patologicheskaya fiziologiya i eksperimental'naya terapiya 2013; 4: 26-31.

13. Onken M., Berger S., Kristian T. Simple model of forebrain ischemia in mouse. J Neurosci Methods 2012; 204(2): 254-261, https://doi.org/10.1016/j.jneumeth.2011.11.022.

14. Tang Y., Wang L., Wang J., Lin X., Wang Y., Jin K., Yang G.Y. Ischemia-induced angiogenesis is attenuated in aged rats. Aging Dis 2016; 7(4): 326, https://doi.org/10.14336/ ad.2015.1125.

15. Pshennikova M.G., Bakhtina L.Yu., Budanova O.P., Malyshev I.Yu. Effect of adaptation to not damaging stress influences on resistance to acute stress in rats August line and Wistar population. Patogenez 2014; 12(4): 31-34.

16. Watanabe A., Sasaki T., Yukami T., Kanki H., Sakaguchi M., Takemori H., Kitagawa K., Mochizuki H. Serine racemase inhibition induces nitric oxide-mediated neurovascular protection during cerebral ischemia. Neuroscience 2016; 339: 139-149, https://doi.org/10.1016/j. neuroscience.2016.09.036.

17. Sheldon R.A., Sadjadi R., Lam M., Fitzgerald R., Ferriero D.M. Alteration in downstream hypoxia gene signaling in neonatal glutathione peroxidase overexpressing mouse brain after hypoxia-ischemia. Dev Neurosci 2015; 37(4-5): 398-406, https://doi.org/10.1159/000375369.
18. Kitagawa K., Matsumoto M., Yang G., Mabuchi T., Yagita Y., Hori M., Yanagihara T. Cerebral ischemia after bilateral carotid artery occlusion and intraluminal suture occlusion in mice: evaluation of the patency of the posterior communicating artery. J Cereb Blood Flow Metab 1998; 18(5): 570-579, https://doi.org/10.1097/00004647-19980500000012.

19. Yang G., Kitagawa K., Ohtsuki T., Kuwabara K., Mabuchi T., Yagita Y., Takazawa K., Tanaka S., Yanagihara T., Hori M., Matsumoto M. Regional difference of neuronal vulnerability in the murine hippocampus after transient forebrain ischemia. Brain Res 2000; 870(1-2): 195-198, https://doi.org/10.1016/s0006-8993(00)02319-2.

20. Wellons J.C. 3rd, Sheng H., Laskowitz D.T., Mackensen G.B., Pearlstein R.D., Warner D.S. A comparison of strain-related susceptibility in two murine recovery models of global cerebral ischemia. Brain Res 2000; 868(1): 14-21, https://doi.org/10.1016/s0006-8993(00)02216-2.

21. Soria F.N., Pérez-Samartín A., Martin A., Gona K.B., Llop J., Szczupak B., Chara J.C., Matute C., Domercq M. Extrasynaptic glutamate release through cystine/glutamate antiporter contributes to ischemic damage. J Clin Invest 2014; 124(8): 3645-3655, https://doi.org/10.1172/jci71886.

22. Beckmann N. High resolution magnetic resonance angiography non-invasively reveals mouse strain differences in the cerebrovascular anatomy in vivo. Magn Reson Med 2000; 44(2): 252-258, https://doi.org/10.1002/15222594(200008)44:2<252::aid-mrm12>3.0.co;2-g.

23. Zhen G., Doré S. Optimized protocol to reduce variable outcomes for the bilateral common carotid artery occlusion model in mice. J Neurosci Methods 2007; 166(1): 73-80, https://doi.org/10.1016/j.jneumeth.2007.06.029.

24. Hermann D.M., Zechariah A., Kaltwasser B., Bosche B., Caglayan A.B., Kilic E., Doeppner T.R. Sustained neurological recovery induced by resveratrol is associated with angioneurogenesis rather than neuroprotection after focal cerebral ischemia. Neurobiol Dis 2015; 83: 16-25, https://doi. org/10.1016/j.nbd.2015.08.018.

25. Katsuragi S., Ikeda T., Ikenoue T. A strategy to treat neonatal hypoxic encephalopathy using glial cell line-derived neurotrophic factor. No To Hattatsu 2011; 43(4): 265-272.

26. Cheng H., Fu Y.-S., Guo J.-W. Ability of GDNF to diminish free radical production leads to protection against kainate-induced excitotoxicity in hippocampus. Hippocampus 2004; 14(1): 77-86, https://doi.org/10.1002/hipo.10145.

27. Wang E., Gao J., Yang Q., Parsley M.O., Dunn T.J., Zhang L., DeWitt D.S., Denner L., Prough D.S., Wu P. Molecular mechanisms underlying effects of neural stem cells against traumatic axonal injury. J Neurotrauma 2012; 29(2): 295-312, https://doi.org/10.1089/neu.2011.2043.

28. Liao Y., Zhong D., Kang M., Yao S., Zhang Y., Yu.Y. Transplantation of neural stem cells induced by all-transretinoic acid combined with glial cell line derived neurotrophic factor and chondroitinase ABC for repairing spinal cord injury of rats. Zhongguo Xiu Fu Chong Jian Wai Ke Za Zhi 2015; 29(8): 1009-1015.

29. Chao M.V. Intercellular networks underlying developmental decisions. Neuron 2016; 91(5): 947-949, https://doi.org/10.1016/j.neuron.2016.08.025.

30. Costantini F., Shakya R. GDNF/Ret signaling and the development of the kidney. BioEssays 2006; 28(2): 117-127, https://doi.org/10.1002/bies.20357.

31. Shang J., Deguchi K., Yamashita T., Ohta Y., 
Zhang H., Morimoto N., Liu N., Zhang X., Tian F., Matsuura T., Funakoshi H., Nakamura T., Abe K. Antiapoptotic and antiautophagic effects of glial cell line-derived neurotrophic factor and hepatocyte growth factor after transient middle cerebral artery occlusion in rats. J Neurosci Res 2010; 88(10): 2197-2206, https://doi.org/10.1002/jnr.22373.
32. Yamashita T., Abe K. Recent progress in therapeutic strategies for ischemic stroke. Cell Transplantation 2016; 25(5): 893-898, https://doi.org/10.3727/096368916x690548.

33. Szydlowska K., Tymianski M. Calcium, ischemia and excitotoxicity. Cell Calcium 2010; 47(2): 122-129, https://doi. org/10.1016/j.ceca.2010.01.003. 